\title{
Article \\ Phylogenetic, Functional and Safety Features of 1950s B. infantis Strains
}

\author{
Stéphane Duboux ${ }^{1, *}$, Catherine Ngom-Bru ${ }^{1}$, Florac De Bruyn ${ }^{2}$ and Biljana Bogicevic ${ }^{1}$ \\ 1 Société des Produits Nestlé SA, Nestlé Research, Route du Jorat 57, CH-1000 Lausanne 26, Switzerland; \\ catherine.ngombru@rdls.nestle.com (C.N.-B.); biljana.bogicevic@rdko.nestle.com (B.B.) \\ 2 Société des Produits Nestlé SA, Nestlé Research \& Development, Nestléstrasse 3, \\ $\mathrm{CH}-3510$ Konolfingen, Switzerland; Florac.debruyn@rd.nestle.com \\ * Correspondence: stephane.duboux@rdls.nestle.com
}

Citation: Duboux, S.; Ngom-Bru, C.; De Bruyn, F.; Bogicevic, B. Phylogenetic, Functional and Safety Features of 1950s B. infantis Strains. Microorganisms 2022, 10, 203. https://doi.org/10.3390/ microorganisms10020203

Academic Editors: Gonzalo Solís Sánchez and Miguel Gueimonde

Received: 25 November 2021

Accepted: 6 January 2022

Published: 18 January 2022

Publisher's Note: MDPI stays neutral with regard to jurisdictional claims in published maps and institutional affiliations.

Copyright: (C) 2022 by the authors. Licensee MDPI, Basel, Switzerland. This article is an open access article distributed under the terms and conditions of the Creative Commons Attribution (CC BY) license (https:// creativecommons.org/licenses/by/ $4.0 /)$.

\begin{abstract}
Strains of Bifidobacterium longum subsp. infantis (B. infantis) are amongst the first to colonize the infant gut, partly due to their capacity to metabolize complex human milk oligosaccharides (HMO), and are proposed to play a key role in the development of the infant gut. Since early life, $B$. infantis supplementation is of high interest, and detailed phylogenetic, functional and safety characterization of the selected strains should be pursued. Using a combination of long and shortread sequencing technologies, we first decipher the genetic distance between different isolates of the same $B$. infantis strain. Using the same approach, we show that several publicly available genomes recapitulate this strain-level distance as compared to two of the first strains obtained in the 1950s. Furthermore, we demonstrate that the two 1950s B. infantis strains display different functional and safety attributes, as ATCC 15697 is resistant to streptomycin and shows a preference towards lacto-Ntetraose LNT and sialylated HMOs, while LMG 11588 is sensitive to all tested antibiotics and shows a preference towards fucosylated HMOs. Overall, our work highlights that the current diversity observed in B. infantis is likely underestimated and that strain selection within this subspecies must be the subject of scientific pursuit and associated evaluation.
\end{abstract}

Keywords: Bifidobacterium; phylogeny; antibiotic resistance

\section{Introduction}

Bifidobacterium spp. present in the human gut microbiome are of key importance for gastrointestinal health throughout the entire human lifespan [1]. This genus is one of the first to establish in the human gastrointestinal tract and is predominant in the neonate gut microbiome until the age of four months [2]. It is proposed that reduced abundance of Bifidobacterium spp. in the gut of infants from developed countries is associated with an increased incidence of allergic and autoimmune diseases later in life [3]. The abundance of B. infantis is in concordance with the general decrease of the Bifidobacterium genus in the developing gut microbiota from infancy to adulthood. While $70 \%$ of breastfed infants from Ghana had detectable levels of $B$. infantis in their gut, no detectable levels could be found in a comparative cohort from both New Zealand and the UK [4]. A more recent study only detected a low prevalence and abundance of $B$. infantis within the faeces of Australian, South-East Asian, and Chinese infants, which contrasted to the relatively high occurrence of B. longum subsp. longum in the same subjects [5].

Strains belonging to $B$. infantis are particularly well-adapted to metabolize the indigestible human milk oligosaccharides (HMOs), which explains at least in part their abundance in the gastrointestinal tract of breast-fed infants [6]. They are also proposed to play a key role in the maturation of the immune system and the improvement of the gut barrier function in early infancy [7]. Altogether, it was hypothesized that restoring a B. infantis-dominant microbiota in early infancy, through the co-administration of HMOs and selected B. infantis strains, is beneficial for the infant immune system maturation [8]. 
The oldest known strains of $B$. infantis available today were isolated in the 1950 s and were initially described as Lactobacillus bifidus [9,10]. Taxonomy of B. longum subsp. infantis has been subjected to several changes and was eventually described and accepted to be a subspecies of the Bifidobacterium longum, for which the type strain is one of the strains isolated by Norris et al in 1950, deposited under ATCC 15697 [11]. The B. longum subsp. infantis strain deposited at the BCCM/LMG collection under LMG 11588 (also deposited at ATCC under ATCC 17930) is another strain isolated by Norris et al., which was substantially less studied than its co-isolated strain. A recent publication shedding light on the diversity of B. longum subsp. infantis showed that the two above-mentioned strains were closely related to several other strains isolated later. If this work suggested a close affiliation between those strains, the analysis performed by Zabel and colleagues was performed using 500 core proteins, which did not enable a strong conclusion on the relationship between the two first described strains, ATCC 15697 and ATCC 17930, and other closely related strains [12].

It has recently been reported that carbohydrate utilization patterns among different strains of B. longum subsp. infantis may vary. Strains belonging to the type strain (ATCC 15697) cluster have been described to consume different types of HMOs simultaneously and equally well with a preference for neutral and sialylated HMOs [6,13-15]. Opposed to that, the Bi-26 strain, clustering together with LMG 11588 (ATCC 17930), was shown to be adapted to rapidly internalize and metabolize fucosylated HMOs [12]. Fucosylated HMOs are highly prevalent in the human breast milk. $2^{\prime}$-fucosyllactose $\left(2^{\prime} \mathrm{FL}\right)$ is the most abundant fucosylated $\mathrm{HMO}$ and represents up to $45 \%$ of the total HMO content of breast milk $[13,16,17]$. The work from Duar et al. suggests that metabolizing neutral and sialylated HMOs equally well presents an advantage, as they showed that the preconditioned EVC001 strain could outcompete the B. longum subsp. infantis NLS strain in vitro and in three human subjects [18]. Contrary to that, it was proposed that metabolizing fucosylated HMOs in preference could provide a competitive advantage in the gut, preventing other microorganisms from accessing this highly abundant substrate present in human breastmilk [12].

Clinical trials assessing safety (as primary or secondary outcomes) of different $B$. longum subsp. infantis strains, namely EVC001 [19] and M-63 [20] are available. However, to date, B. longum subsp. infantis R0033, which clusters together with the LMG 11588 (ATCC 17930) strain [12], and the DSM 33361 strain for which the genome is not publicly available, are the only strains that have been extensively evaluated by regulatory authorities, and have been communicated to the US Food and Drug Administration as generally recognized as safe (GRAS) without objection [21,22].

In this work, we aim to better understand the genetic and phenotypic diversity encompassed within B. longum subsp. infantis. Furthermore, by comparing the characteristics of ATCC 15697 and LMG 11588, as representatives of the two bigger phylogenetic clusters of $B$. longum subsp infantis, we not only confirm that the carbohydrate metabolism of those two groups are different, but as well highlight that their genome-based safety characteristics differ, supporting the overall need for a detailed characterization of strains intended for infant nutrition.

\section{Materials and Methods}

\subsection{Bacterial Strains}

B. longum subsp. infantis type strain isolates were obtained from three different culture collections. The type strain was obtained from the American Type Culture Collection as ATCC 15697 (ATCC, Manassas, VA, USA), from the Belgian Coordinated Collection of Microorganisms as LMG 08811 (BCCM/LMG, Ghent, Belgium) and was retrieved from the Nestlé Culture Collection (NCC) under NCC 341. B. longum subsp. infantis LMG 11588 was obtained from BCCM/LMG. Upon reactivation, strains were stored within the NCC in a freeze-dried powder form. Strains were commonly cultivated in MRSc growth medium (MRS broth supplemented $0.05 \% w / v$ L-Cysteine), in anaerobiosis at $37^{\circ} \mathrm{C}$ for $16 \mathrm{~h}$. 


\subsection{DNA Preparation and Sequencing}

Strains were reactivated from the NCC freeze-dried stocks in MRS broth (Oxoid AG, Pratteln, Switzerland) containing 0.05\% w/v L-Cysteine (Sigma-Aldrich, Darmstadt, Germany). $5 \mathrm{~mL}$ of mid-exponentially grown culture were harvested by centrifugation $(3000 \times g, 5 \mathrm{~min})$ and kept frozen for later DNA preparation. DNA was prepared using the QiaAmp kit (Qiagen GmbH, Hilden, Germany) for Illumina sequencing or with MagAttract HMW DNA Kit (Qiagen GmbH, Hilden, Germany) for PacBio Sequencing.

Illumina sequencing was performed as follows. Libraries were prepared following Nextera XT protocol (Illumina Inc., San Diego, CA, USA) and cleaned using the bead-based AMPure kit (Beckman Coulter Life Sciences, Brea, CA, USA). Quality was evaluated using a LabChip GX touch capillary electrophoresis system (PerkinElmer, Waltham, MA, USA) and DNA was pooled equimolarly according to the lowest sample concentration (between 2 and $10 \mathrm{nM}$ ). Sequencing was performed on a HiSeq 2500 sequencer (Illumina Inc., San Diego, CA, USA), using the v4 chemistry PE125 and dual indexing with index of height bases each. Illumina raw sequencing data obtained for the different isolates of ATCC 15697 (accession numbers SRR17574374, SRR17574375, SRR17574376) and LMG 11588 (accession number SRR17574377) were deposited at the Sequence Read Archive (SRA) hosted by NCBI.

PacBio sequencing was performed according to the supplier's recommendation. The DNA library preparation was performed following the recommended protocol from PacBio: "Preparing multiplexed microbial library using the SMRTbell Express Template Prep Kit 2.0" (Part Number 101-696-100 V.07 (July 2020)). DNA quality was checked along the library preparation using the Fragment Analyzer (Agilent Technologies, Inc., Santa Clara, CA, USA) and quantified by QuBit dsDNA protocol (Thermo Fisher Scientific AG, Basel, Switzerland). Sequencing was performed on a Sequel platform with $10 \mathrm{~h}$ movies on an LR SMRT cell. The loading was performed by diffusion at 8:00 p.m. with $2 \mathrm{~h}$ of pre-extension time. The sequencing data was further assembled using the Hierarchical Genome Assembly Process (HGAP4) de novo assembly analysis application available through the SMRT Link portal (Pacific Biosciences, Menlo Park, CA, USA). The obtained PacBio assembled genome of LMG 11588 was deposited at the Joint Genome Institute (JGI) under Project ID Ga0526375.

\subsection{Average Nucleotide Identity Based Phylogeny}

All non-sequenced genomes were retrieved from public repositories (Supplementary Table S1). Average Nucleotide Identity (ANI) was computed using the OrthoANIu software (v.1.2, https:/ / www.ezbiocloud.net/tools/orthoaniu, accessed on 12 February 2021) to compare genome assemblies [23]. The generated matrix of pairwise genome similarities (Supplementary Table S2) was further used to build a phylogenetic tree. To assess the validity of clusters of interest, $p$-values were calculated for hierarchical clustering via multiscale bootstrap resampling ( $n=10^{4}$ replications) with $\mathrm{H}_{0}$ (null hypothesis) $=$ cluster is not supported by data and $\mathrm{H}_{1}$ (alternative hypothesis) = cluster is supported by data at significance level $\alpha=0.05$.

\subsection{Single Nucleotide Polymorphism Comparisons}

A high-quality single nucleotide polymorphism (SNP) pipeline developed by the Center for Food Safety and Applied Nutrition (CFSAN SNP Pipeline v.1.0.0/FDA) [24] was used for SNP calling [25]. Publicly available genomes were used as references to map short read sequencing data obtained from LMG 11588 or from the different ATCC 15697 isolates.

\subsection{Genome Based Safety Analysis}

Putative virulence genes of $B$. longum subsp. infantis LMG 11588 and ATCC 15697 were searched by BLAST analysis against the publicly available VFDB [24]. Putative antibiotic resistance genes of B. infantis LMG 11588 and ATCC 15697 were searched for using ResFinder [25], PointFinder [26] (both software and associated databases downloaded on from https: / /bitbucket.org/genomicepidemiology/, accessed on 2 December 2019) and AMRFinder (v.3.10, https://github.com/ncbi/amr/wiki, accessed on 15 March 2021) [27]. 
Putative enzymes resulting in the production of harmful metabolites were searched for using BLAST analysis (v.2.5.0, ftp:/ / ftp.ncbi.nlm.nih.gov/blast/executables/blast+, accessed on 15 March 2021) against previously identified genes in the B. longum JDM301 strain [28]. As the B. longum JDM301 strain has no enzyme involved in the production of biogenic amines, protein sequences of such enzymes were searched through the MetaCyc web site (https: / / metacyc.org, accessed on 15 September 2021) [29]. Protein sequences from bacteria for histidine decarboxylase (EC 4.1.1.22; P28577, P00862), lysine decarboxylase (EC 4.1.1.18; B6EP92, O50657, Q9L072, P0A9H3, P52095) and ornithine decarboxylase (EC 4.1.1.17; P21169, P24169) could be retrieved and used for BLAST analysis. Only hits with percent identity $>70 \%$ and coverage $>60 \%$ were considered as significant for all readouts and reported.

\subsection{HMO Consumption Profiles}

Bifidobacterium longum subsp. infantis strains LMG 11588 and ATCC 15697 were resuscitated in a basal medium (containing yeast-derived amino acids at $3 \%$ and vitamin C) with glucose as the sole carbon source (anaerobic $37^{\circ} \mathrm{C}$ for $18 \mathrm{~h}$ ) and then transferred into the same medium, replacing glucose with a mixture of five HMOs a sole carbon source (aerobically $37^{\circ} \mathrm{C}$ for $24 \mathrm{~h}$ ). The HMOs were added at a total concentration of $2 \% w / w$ with ratios representing human breast milk composition, respectively: $2^{\prime}$-fucosyllactose $\left(2^{\prime} \mathrm{FL}\right) 61 \%$, lacto-N-tetraose (LNT) $20 \%, 6^{\prime}$-sialyllactose (6'SL) $9 \%$, difucosyllactose (diFL) $8 \%, 3^{\prime}$-sialyllactose ( $\left.3^{\prime} \mathrm{SL}\right) 2 \%$. All media were autoclaved at $121^{\circ} \mathrm{C}$ for $15 \mathrm{~min}$. Carbohydrate solutions were filter sterilized $(20 \mu \mathrm{M}$ average pore diameter) and added aseptically after autoclavating. Medium $\mathrm{pH}$ was monitored in real-time as a growth measure. Initial and residual HMO concentrations were quantified by liquid chromatography with fluorescence detection after labelling with 2-aminobenzamide using the protocol described earlier [30]. All HMOs were quantified against genuine analytical standards with known purity. All carbohydrates and analytical standards were obtained from Glycom A/S (Hørsholm, Denmark).

\subsection{Phenotypic Antibiotic Resistance Profiling by Microdilution}

Phenotypic antibiotic testing of the B. longum subsp. infantis strains was performed according to the recommendations made by EFSA [31] following the official ISO 10932 method. In short, bacterial strains were propagated on MRSc agar plates and grown for $48 \mathrm{~h}$ at $37^{\circ} \mathrm{C}$ in anaerobiosis. Colonies obtained were then resuspended (McFarland turbidity of 1) in $2 \mathrm{~mL}$ of physiological saline buffer. Ten (10) $\mu \mathrm{L}$ of this solution were then used to inoculate $10 \mathrm{~mL}$ of LSM broth (for $1000 \mathrm{~mL}: 21.06 \mathrm{~g}$ of Iso-SensiTest broth (Thermo Fisher Scientific AG, Basel, Switzerland); 5.2 g of MRS broth (Oxoid AG, Pratteln, Switzerland) supplemented with $0.05 \%$ of cysteine (Sigma-Aldrich, Darmstadt, Germany) having a final $\mathrm{pH}$ of 6.7. Hundred (100) $\mu \mathrm{L}$ of inoculated medium were then transferred in each well of precoated Sensititre ${ }^{\mathrm{TM}}$ antibiotic plates (Thermo Fisher Scientific AG, Basel, Switzerland), which were finally incubated for $48 \mathrm{~h}$, at $37^{\circ} \mathrm{C}$, in anaerobic condition. Minimal inhibitory concentrations (MIC) were determined by reading the turbidity in each well after $48 \mathrm{~h}$ of incubation and were compared to the thresholds determined by EFSA for antibiotics of human and veterinary importance.

\section{Results}

\subsection{Phylogeny of the B. longum subsp. infantis Subspecies}

All publicly available genomes of $B$. longum subsp. infantis that had been previously compared using 500 core proteins [12] were retrieved and compared by average nucleotide identity (ANI), employing their full genomic information (Figure 1 ). The publicly available genome of B. longum subsp. longum NCC 2705 was added as an outlier, and clustered separately $(95.040 \%$ ANI) to all B. longum subsp. infantis strains. A comparison of all B. longum subsp. infantis strains showed that several clearly distinguishable strains exist (UBBI-01, 2_mod, IN-F29, TPY12-1); all other strains being regrouped in three different 
clusters. A first cluster consisting of R0033, Bi-26 and EK3 share a mean homology of $>99.960 \%$ ANI to the LMG11588 (ATCC 17930) strain, one of the first described isolates of B. longum subsp. infantis [9]. The second cluster, sharing $98.280 \%$ ANI with the first one, contains a set of strains, including NCTC13219 and EVC001, which were all shown to be closely related (mean homology $>99.993 \%$ ANI) to the ATCC 15697 type strain, which was also one of the first described isolates of B. longum subsp. infantis [9]. These two clusters can also be differentiated from each other based on their average genome size and average GC content. Indeed, the LMG 11588 genome cluster has an average genome size of 2,599,193 bp and average GC content of 58.97\%, while ATCC 15697 genome cluster has higher average genome size and average GC content (respectively 2,795,592 bp and $59.60 \%$ ). Finally, the strains $1888 \mathrm{~B}$ and BT1 were shown to be closely related to each other $(>99.993 \%$ ANI) (Figure 1). These three clusters are supported by bootstrapping with $p<0.05$ (Supplementary Figure S1).

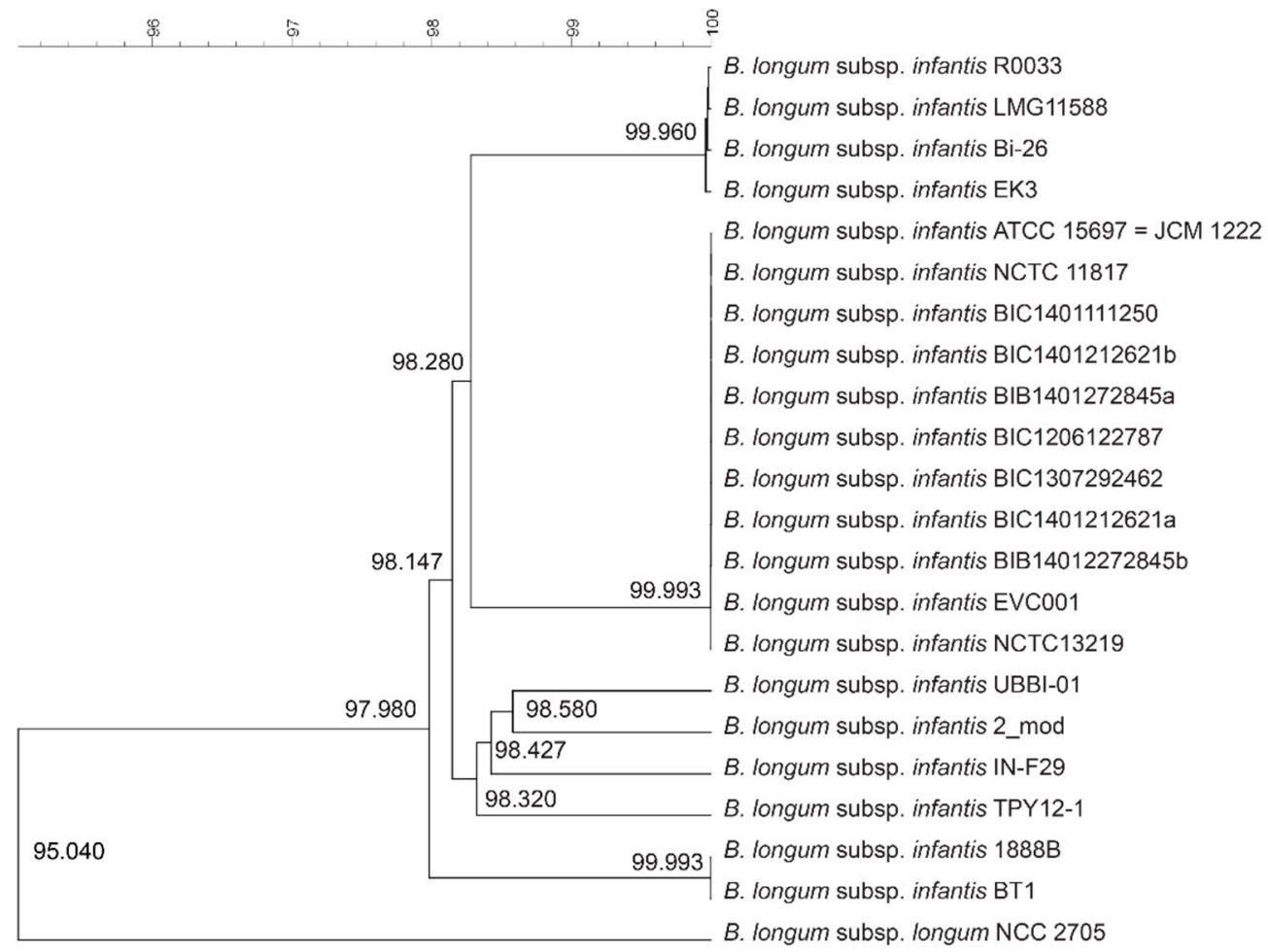

Figure 1. Whole genome based phylogenetic UPGMA tree of publicly available $B$. longum subsp. infantis strains. The genome of B. longum subsp. longum NCC 2705 was used as an outlier. Average Nucleotide Identity percentage (\% ANI) is depicted at each branch node.

\subsection{SNP Variation Observed in Different Isolates of B. infantis ATCC 15697}

To determine the genetic variation that can be observed within different isolates of the same strain of B. longum subsp. infantis, we sourced the ATCC 15697 type strain from different culture collections, sequenced the obtained isolates and compared them to publicly available genome sequences of this same strain using the SNP comparison pipeline developed by the US-FDA. Using this pipeline, a threshold of $<20$ SNPs, associated with a monophyletic phylogenetic tree topology was suggested to ensure that the pathogenic isolates are from "the same origin" [32]. Analysis showed that the number of SNPs ranges from a minimum of four up to a maximum of 15 between different isolates and sequences of the B. infantis type strain (Table 1 ). 
Table 1. Genomic SNP level comparison of different isolates of the B. infantis ATCC 15697 (type strain) sourced from different culture collection. All three isolates were compared to the two publicly available genomes of the strain. Genome accession numbers and sizes are indicated in parentheses.

\begin{tabular}{cccc}
\hline Public Genome Version & LMG 08811 & ATCC 15697 & NCC 341 \\
\hline ATCC 15697 (NC_017219.1; 2,828,958 bp) & 5 & 4 & 13 \\
ATCC 15697 (NC_011593.1; 2,832,748 bp) & 8 & 14 & 15 \\
\hline
\end{tabular}

\subsection{Relationship of B. infantis ATCC 15697 and LMG 11588 to Other Closely Related Strains}

To better understand the relationship of strains found to be closely related to ATCC 15697 and LMG 11588, the two initial strains of B. longum subsp. infantis described in the 1950s [9], we compared them all at SNP level, as described previously. We found that all the strains showing an ANI above $99.9 \%$ to ATCC 15697 displayed no more than 32 SNPs to that strain. Out of the 10 strains encompassed in this cluster, four strains displayed levels similar (0-16 SNPs) to what we observed previously for different isolates of the same strain. The other six strains displayed a slightly higher number of polymorphisms (26-32 SNPs) (Table 2). Similarly, all strains showing an ANI of more than 99.9\% to LMG 11588 displayed not more than 13 SNPs to that strain (Table 3).

Table 2. Number of SNPs found between the B. infantis ATCC 15697 sequenced strain and closely related publicly available genomes (ANI > 99.9\%).

\begin{tabular}{cc}
\hline Strain Name (Genome Size in Base Pair) & ATCC 15697 \\
\hline NCTC13219 $(2,602,591 \mathrm{bp})$ & 0 \\
NCTC11817 $(2,832,748 \mathrm{bp})$ & 5 \\
BIB1401272845a $(2,791,524 \mathrm{bp})$ & 13 \\
BIC1401212621a $(2,791,569 \mathrm{bp})$ & 31 \\
BIC1401212621b $(2,821,883 \mathrm{bp})$ & 16 \\
BIB1401272845b (2,786,838 bp) & 30 \\
Strain_6 (EVC001) (2,832,850 bp) & 28 \\
BIC1206122787 (2,789,037 bp) & 26 \\
BIC1401111250 (2,793,888 bp) & 33 \\
BIC1307292462 (2,879,623 bp) & 32 \\
\hline
\end{tabular}

Table 3. Number of SNPs found between the B. infantis LMG 11588 sequenced strain and closely related publicly available genomes (ANI > 99.9\%).

\begin{tabular}{cc}
\hline Strain Name (Genome Size in Base Pair) & LMG 11588 \\
\hline $\operatorname{R0033}(2,615,717 \mathrm{bp})$ & 16 \\
$\operatorname{Bi}-26(2,569,437 \mathrm{bp})$ & 4 \\
$\operatorname{Ek3}(2,564,809 \mathrm{bp})$ & $13\left(231^{1}\right)$ \\
\hline
\end{tabular}

${ }^{1}$ SNPs identified in genomic regions where stretches of undetermined nucleotides ("N") were present.

A total of 231 "raw" SNPs were identified when mapping the LMG 11588 reads against the EK3 strain genome assembly. When examining all SNPs, it was found that a lot of clustered SNPs were identified in regions of the EK3 strain genome assembly where stretches of undetermined nucleotides (" $\mathrm{N}$ ") were present. These regions were considered as unreliable, and associated SNPs were removed, resulting in a final count of 13 SNPs difference between the EK3 and LMG 11588 strains.

\subsection{HMO Consumption Profiles of B. infantis ATCC 15697 and LMG 11588}

It was previously shown that two HMO consumption patterns exist among strains of B. longum subsp. infantis [12-15]. To determine if the two strains isolated in the 1950s (ATCC 15697 and LMG 11588) reflect those differences, both strains were cultured for $24 \mathrm{~h}$ in a medium containing a mix of five HMOs as the sole carbon source. Consumption of each individual $\mathrm{HMO}$ was measured in the culture supernatant using liquid chromatography 
with fluorescence detection. Results showed that the type strain ATCC 15697 consumed the entire LNT pool present in the mix $(-100 \%)$, had a preference towards sialylated HMOs (3'SL $\left.-67 \% ; 6^{\prime} \mathrm{SL}-43 \%\right)$, and consumed the fucosylated HMOs to a lower degree $\left(2^{\prime} \mathrm{FL}\right.$, $-23 \%$; diFL, $-21 \%$ ) (Figure 2). The observed consumption profile of the ATCC 15697 strain reflects previously described ones for this strain and other closely related strains (e.g., EVC001) [18].
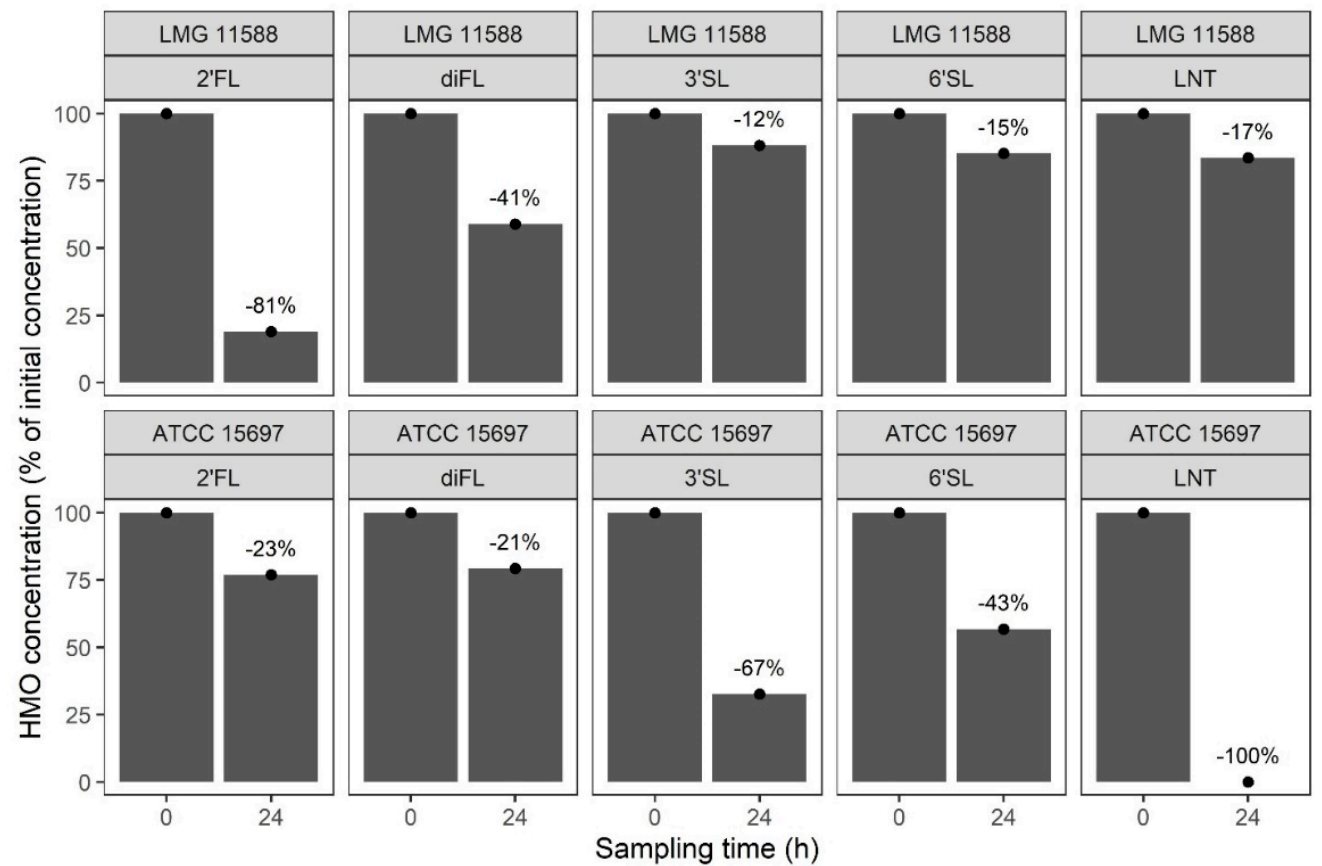

Figure 2. HMO consumption profile of $B$. infantis ATCC 15697 and LMG 11588 grown for $24 \mathrm{~h}$ in a medium containing a mix of five HMOs as sole carbon source.

In contrast, the LMG 11588 strain preferably consumed both fucosylated HMOs present in the mix $\left(2^{\prime} \mathrm{FL},-81 \%\right.$; diFL, $\left.-41 \%\right)$ and metabolized other HMOs present to different degrees (LNT, $-17 \% ; 3^{\prime} \mathrm{SL},-12 \% ; 6^{\prime} \mathrm{SL},-15 \%$ ) (Figure 2). This efficient consumption of fucosylated HMOs has already been described previously for another closely related strain (Bi-26) [12].

3.5. Phenotypic Antibiotic Resistance Profiles and rpSL Gene Mutation in B. infantis ATCC 15697 and LMG 11588

Antibiotic resistance was assessed using the microdilution method. Obtained minimal inhibitory concentrations (MICs) were compared to the cut-off determined by EFSA. Analysis showed that $B$. infantis LMG 11588 was sensitive to all antibiotics tested, while the type strain B. infantis ATCC 15697 was resistant to streptomycin since the MIC obtained for this antibiotic was of $512 \mu \mathrm{g} / \mathrm{mL}$ and higher than the resistance cut-off determined by EFSA (Table 4).

The observed streptomycin resistance in the ATCC 15697 strain could be explained by the presence of a point mutation in the $r p S L$ gene of this strain (A to $\mathrm{G}$ replacement occurring at nucleotide 128) (Table 5). This mutation was initially described in B. breve [33] and was also detected in several Bifidobacterium strains, including B. longum subsp. infantis M-63 [34]. While screening the LMG 11588 strain genome, the mer $(A)$ gene was identified. This gene was not found in the ATCC 15697 genome. Mercury resistance induced by mer genes was initially suspected to be positively associated with multidrug resistance in Gram negative human fecal bacteria [35]. However, this association could not be shown for Gram positive bacteria [36]. 
Table 4. Phenotypic resistance of B. infantis ATCC 15697 and LMG 11588 to relevant antibiotics as determined by EFSA. Minimal inhibitory concentrations (MICs) were determined using the microdilution method and are expressed in $\mu \mathrm{g} / \mathrm{mL}$.

\begin{tabular}{cccc}
\hline Antibiotic & ATCC 15697 & LMG 11588 & Cut-Off Determined by EFSA \\
\hline Gentamicin $(\mu \mathrm{g} / \mathrm{mL})$ & 1.0 & 32.0 & 64.00 \\
Streptomycin $(\mu \mathrm{g} / \mathrm{mL})$ & 512.0 & 16.0 & 128.00 \\
Tetracyclin $(\mu \mathrm{g} / \mathrm{mL})$ & 2.0 & 2.0 & 8.00 \\
Erythromycin $(\mu \mathrm{g} / \mathrm{mL})$ & 0.125 & 0.5 & 1.00 \\
Clindamycin $(\mu \mathrm{g} / \mathrm{mL})$ & 0.125 & 0.125 & 1.00 \\
Chloramphenicol $(\mu \mathrm{g} / \mathrm{mL})$ & 1.0 & 2.0 & 4.00 \\
Ampicillin $(\mu \mathrm{g} / \mathrm{mL})$ & 0.13 & 0.25 & 2.00 \\
Vancomycin $(\mu \mathrm{g} / \mathrm{mL})$ & 1.0 & 0.5 & 2.00 \\
\hline
\end{tabular}

Table 5. rpSL gene sequence at position 128 (of B. breve rpSL gene).

\begin{tabular}{ccc}
\hline Strain & Streptomycin Resistance & rpSL Gene, Positions 122-134 \\
\hline B. breve strain Yakult [33] & resistant & cccogaGgaagcc \\
B. infantis ATCC 15697 & resistant & cccogaGgaagcc \\
B. infantis LMG 11588 & sensitive & cccogaAgaagcc \\
\hline
\end{tabular}

3.6. Genetic Organization around Mutated rpSL Gene in B. infantis ATCC 15697 and LMG 11588

The genome of ATCC 15697 revealed that the mutated rpSL gene (Blon_1925) was encompassed by different transposases. Two IS110 family proteins were found in the close vicinity (Blon_1914 and Blon_1928) which shared a relatively low level of identity to each other (48\%). Two ISBlo11 family transposases (Blon_1909 and Blon_1931) with an identity of $100 \%$ encompassing the full genomic region were found further away. Even if the two ISBlo11 transposases are oriented in the same direction, they contain inverted repeats typical of this transposase family, suggesting that they are fully functional. Supporting this, ISblo11 transposases were previously found to be fully functional, and were even proposed to be employed for a transposon-mediated mutagenesis system in bifidobacteria [37]. The organization of genes surrounding the rpSL gene of LMG 11588 (KY279_001650) was found to be slightly different. An IS21 type transposase and its IS21-like element helper ATPase (KY279_001655 and KY279_001654 respectively) are located downstream of the $r p S L$ gene, but were found to be frameshifted. Upstream of the rpSL gene of LMG 11588, a frameshifted transposase (KY279_001639), an integrase from the IS3 family (KY279_001638), and a relaxase (KY279_001637) were also found (Figure 3).

\subsection{Presence of Other Safety Related Genes}

We screened both LMG 11588 and ATCC 15697 genomes for the presence of virulence encoding genes as well as genes encoding enzymes responsible for the production of potentially harmful metabolites. No genes related to the production of virulence factors could be detected in both genomes. Genes encoding conjugated bile salt hydrolase (CBSH), Dlactate dehydrogenase (DLD) and nitroreductase (ND) could be observed in both genomes. Beta-glucuronidase genes could be detected independently in the JDM301 (BLJ_0623) and LMG 11588 (KY279_001620), but not in ATCC 15697 genome (Table 6). No genes known to code for tyrosine decarboxylase, ornithine decarboxylase, histidine decarboxylase, lysine decarboxylase, arylsulfatase or azoreductase could be found in any of the LMG 11588 and ATCC 15697 genomes. 

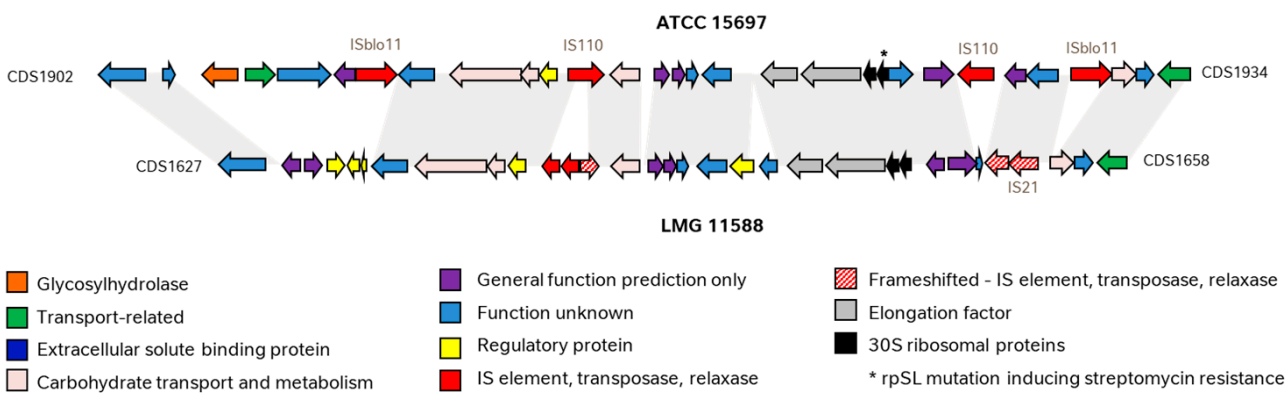

Figure 3. Comparison of the genomic organization surrounding the rpSL gene of B. longum subsp. infantis ATCC 15697 and LMG 11588. Homologous DNA portions are indicated in grey. Functional classes are represented by different colors.

Table 6. Significant BlastP hits for putative enzymes implicated in the production of harmful metabolites in B.infantis ATCC 15697 and LMG 11588 genomes.

\begin{tabular}{cccc}
\hline Enzymes & JDM301 Locus_Tag & $\begin{array}{c}\text { LMG 11588 Locus_Tag } \\
\text { (\% Identity; Coverage) }\end{array}$ & $\begin{array}{c}\text { ATCC 15697 Locus_Tag } \\
\text { (\% Identity; Coverage) }\end{array}$ \\
\hline $\begin{array}{c}\text { Conjugated bile salt hydrolase (CBSH) } \\
\text { D-lactate dehydrogenase (DLD) }\end{array}$ & BLJ_0948 & KY279_001316 (98.74; 0.99) & Blon_1453 (98.42; 1) \\
& BLJ_1306 & KY279_000900 (98.50; 1) & Blon_0845 (98.50; 1) \\
Nitroreductase (NR) & BLJ1436 & KY279_000777 (96.95; 1) & Blon_0718 (97.26; 1) \\
\hline
\end{tabular}

\section{Conclusions and Discussion}

According to our whole genome comparative analysis, a significant number of $B$. infantis strains described today are falling into two distinct clusters, which are grouped around two isolates obtained in the 1950s: ATCC 15697 and LMG 11588 (ATCC 17930). All strains belonging to the same cluster are sharing an average nucleotide identity (ANI) of more than $99.9 \%$ with one of those two strains, and most of them displayed a low number of SNPs $(<21)$ compared to one of the two isolates from the 1950s. The SNP analysis pipeline used in this work has been developed by UCFSAN-FDA to determine and track the source of different foodborne contaminating isolates. Based on several outbreak investigations, it was considered that isolates sharing fewer than 21 SNPs over their whole genome can be considered as originating from the same source [32]. Our analysis revealed a maximum of 15 SNPs in several isolates of the same ATCC 15697 strain, representing the expected genetic variation displayed in a single strain of B. longum subsp. infantis. These differences may be caused by the strain propagation or simply be the result of different sequencing and assembly procedures.

Our results demonstrate that the two oldest known isolates of B. longum subsp infantis (ATCC 15697 and LMG 11588) are closely related to several of the strains that have been isolated and sequenced in the last decades, suggesting that the strains organized in the two corresponding clusters are highly clonal. Those results partially repeat a situation already observed within different strains of B. animalis subsp. lactis [38]. Even if several publicly available genomes share strain-level genetic distance to the two strains initially isolated by Norris et al, it is worth noting that, through evolution, several of those strains might have diverged phenotypically, as a few distinct SNPs in coding or regulatory regions might create phenotypic differentiation [39]. Further research is therefore required to understand the potential implications of SNP level differences within the two aforementioned clusters.

An important feature studied in B. infantis strains is their adaption to use HMOs in a very particular way. Until recently, the HMO consumption patterns displayed by B. infantis strains were believed to be relatively homogenous, consuming neutral and sialylated HMOs simultaneously and equally well $[6,13-15,18]$. Recently, Zabel and colleagues demonstrated that members of this subspecies exhibit distinct metabolic profiles, showing that the Bi-26 strain had a strong preference for fucosylated HMOs [12], suggesting the existence of a wider diversity in the B. infantis subspecies. 
Our results on HMO consumption patterns of both ATCC 15697 and LMG 11588 are consistent with previous observations made for some strains belonging to the same cluster. We demonstrated that when grown on a mix of several 5 HMOs (2'FL, diFL, LNT, 3'SL and 6'SL), ATCC 15697 consumes LNT particularly well, but also consumes other sialylated HMO, confirming findings obtained previously on the same strain [6,13-15], and a closely related strain, namely EVC001 ( $>99.9 \%$ ANI) [18]. Similarly, the obtained HMO consumption profile of LMG 11588, showing a preference towards fucosylated HMOs, has also been observed for the closely related Bi-26 strain [12]. As can be expected from their close genetic relationship (>99.9\% ANI), LMG 11588 and Bi-26 have the exact same HMO utilization gene setups. It is therefore interesting that despite the fact that LMG 11588 displays a non-functional ABC transporter system predicted to import LNT (Blon_2175 to Blon_2177 in ATCC 15697) [12], it is still able to partly metabolize LNT, suggesting the existence of a potential secondary LNT transport mechanism in that strain, which is today still to be described. On the other hand, Zabel and colleagues have demonstrated in Bi-26 that expression of different fucose utilization genes were upregulated in the presence of $2^{\prime} \mathrm{FL}, 3^{\prime} \mathrm{FL}$ or diFL, which could partly explain the efficient utilization of those substrates by the close relative LMG 11588 [12].

The antibiotic resistance profile of strains that are intentionally added to food or feed is today particularly scrutinized by regulatory authorities [31,40]. The increase in antibiotic resistance around the globe represents a serious public health challenge. Potentially transferable antibiotic resistances should particularly be avoided, as it has been hypothesized that this could represent a reservoir of resistance and could be transferred to other bacteria present in the gastro-intestinal tract [41]. The two above-described B. longum subsp. infantis strains also differ in this respect. Our phenotype analysis exposed streptomycin resistance in B. infantis ATCC 15697 as described by Kim and colleagues [42], but not in LMG 11588 . B. infantis ATCC 15697, and closely related strains harbor a mutation in the 30S ribosomal gene $r p S L$ that was previously shown to encode a streptomycin resistance in $B$. breve [33] and was shown to be present in other bifidobacterial strains, including B. longum subsp. infantis M-63 [34]. In the presence of streptomycin, the $r p S L$ mutation conferring resistance occurred at a relatively high frequency $\left(10^{-8}\right.$ to $\left.10^{-9}\right)$ [33]. Our analysis of the region surrounding the mutated rpSL gene in ATCC 15697 showed the presence of transposases, of which at least two are likely functional. Similar IS elements (ISBlo11) have been shown to be functional and even proposed to be employed for the development of a transposonmediated mutagenesis system in bifidobacteria [37], suggesting that this region in ATCC 15697 and other B. infantis strains from the same cluster might be transferable to other microorganisms. On the other side, the gene setup surrounding non-mutated $r p S L$ genes of $B$. infantis LMG 11588 should not be of concern. First, the rpSL gene does not harbor the mutation encoding streptomycin resistance. Second, the transposases surrounding it should not enable transfer of the region as they are from different families (IS3 and IS21) and partly non-functional (frameshifted). Today, streptomycin is still an actively used antibiotic [43]. Hence, further studies are required to assess the transferability of this genetic region in B. infantis ATCC 15697 and closely related strains and clarify the potential safety issue this might represent.

Overall, our results highlight that the current diversity observed in B. infantis is likely underestimated. Indeed, the genetic diversity observed in a number of publicly available strains suggest that they are clonally related. We further showed that all the closely related strains described in this work are regrouped in two distinct clusters, each of them containing one of the two first isolated B. infantis strains obtained in the 1950s by Norris et al., ATCC 15697 and LMG 11588, respectively. Finally, we can confirm that those two representative strains harbor different functional and safety attributes, highlighting the need for a detailed strain evaluation when developing commercial B. infantis products intended for early life supplementation. 
Supplementary Materials: The following supporting information can be downloaded at: https: / / www.mdpi.com/article/10.3390/microorganisms10020203/s1, Figure S1: Average nucleotide difference with bootstrapping $p$-values for clusters of interest, Table S1: List of B. longum subsp. infantis strains and genomes used in this study, Table S2: Average Nucleotide Identity (\% ANI) matrix of compared genomes.

Author Contributions: Conceptualization, S.D. and B.B.; methodology, S.D., F.D.B. and C.N.-B.; software, C.N.-B.; formal analysis, S.D., F.D.B. and C.N.-B.; resources, B.B.; writing-original draft preparation, S.D.; writing-review and editing, S.D., F.D.B., C.N.-B. and B.B.; visualization, S.D. and F.D.B. All authors have read and agreed to the published version of the manuscript.

Funding: This research was funded by Société des Produits Nestlé SA.

Institutional Review Board Statement: Not applicable.

Informed Consent Statement: Not applicable.

Data Availability Statement: All assembled B. longum subsp. infantis genomes used in this study are listed in Supplementary Table S1. Raw sequencing data used for the reported SNP analysis have been deposited at the Sequence Read Archive (SRA) hosted by NCBI (accession numbers SRR17574374, SRR17574375, SRR17574376 and SRR17574377). The annotated PacBio LMG 11588 genome is deposited at the JGI under Project ID Ga0526375.

Acknowledgments: We thank Sean Austin and Thierry Benet for the support given regarding HMO quantifications. We thank Anne Bruttin for execution of antibiotic microdilution assays and Solenn Pruvost for the sequencing of the strains used in this work.

Conflicts of Interest: All authors are employed by Société des Produits Nestlé SA.

\section{References}

1. Arboleya, S.; Watkins, C.; Stanton, C.; Ross, R.P. Gut Bifidobacteria Populations in Human Health and Aging. Front. Microbiol. 2016, 7, 1204. [CrossRef] [PubMed]

2. Avershina, E.; Storrø, O.; Øien, T.; Johnsen, R.; Wilson, R.; Egeland, T.; Rudi, K. Bifidobacterial Succession and Correlation Networks in a Large Unselected Cohort of Mothers and Their Children. Appl. Environ. Microbiol. 2013, 79, 497-507. [CrossRef] [PubMed]

3. Henrick, B.; Hutton, A.A.; Palumbo, M.C.; Casaburi, G.; Mitchell, R.D.; Underwood, M.A.; Smilowitz, J.T.; Frese, S.A. Elevated Fecal pH Indicates a Profound Change in the Breastfed Infant Gut Microbiome Due to Reduction of Bifidobacterium over the Past Century. mSphere 2018, 3, e00041-18. [CrossRef]

4. Young, S.L.; Simon, M.A.; Baird, M.A.; Tannock, G.W.; Bibiloni, R.; Spencely, K.; Lane, J.M.; Fitzharris, P.; Crane, J.; Town, I.; et al Bifidobacterial Species Differentially Affect Expression of Cell Surface Markers and Cytokines of Dendritic Cells Harvested from Cord Blood. Clin. Vaccine Immunol. 2004, 11, 686-690. [CrossRef]

5. Lawley, B.; Munro, K.; Hughes, A.; Hodgkinson, A.J.; Prosser, C.G.; Lowry, D.; Zhou, S.J.; Makrides, M.; Gibson, R.A.; Lay, C.; et al. Differentiation of Bifidobacterium longum subspecies longum and infantis by quantitative PCR using functional gene targets. Peer] 2017, 5, e3375. [CrossRef]

6. Garrido, D.; Dallas, D.; Mills, D.A. Consumption of human milk glycoconjugates by infant-associated bifidobacteria: Mechanisms and implications. Microbiology 2013, 159, 649-664. [CrossRef] [PubMed]

7. Underwood, M.A.; German, J.B.; Lebrilla, C.B.; Mills, D.A. Bifidobacterium longum subspecies infantis: Champion colonizer of the infant gut. Pediatr. Res. 2015, 77, 229-235. [CrossRef] [PubMed]

8. Insel, R.; Knip, M. Prospects for primary prevention of type 1 diabetes by restoring a disappearing microbe. Pediatr. Diabetes 2018, 19, 1400-1406. [CrossRef] [PubMed]

9. Norris, R.F.; Flanders, T.; Tomarelli, R.M.; Gyorgy, P. The isolation and cultivation of Lactobacillus bifidus; a comparison of branched and unbranched strains. J. Bacteriol. 1950, 60, 681-696. [CrossRef]

10. Pine, L.; Howell, A. Comparison of Physiological and Biochemical Characters of Actinomyces spp. with those of Lactobacillus bifidus. J. Gen. Microbiol. 1956, 15, 428-445. [CrossRef] [PubMed]

11. Mattarelli, P.; Bonaparte, C.; Pot, B.; Biavati, B. Proposal to reclassify the three biotypes of Bifidobacterium longum as three subspecies: Bifidobacterium longum subsp. longum subsp. nov., Bifidobacterium longum subsp. infantis comb. nov. and Bifidobacterium longum subsp. suis comb. nov. Int. J. Syst. Evol. Microbiol. 2008, 58, 767-772. [CrossRef]

12. Zabel, B.E.; Gerdes, S.; Evans, K.C.; Nedveck, D.; Singles, S.K.; Volk, B.; Budinoff, C. Strain-specific strategies of 2'-fucosyllactose, 3-fucosyllactose, and difucosyllactose assimilation by Bifidobacterium longum subsp. infantis Bi-26 and ATCC 15697. Sci. Rep. 2020, 10, 15919. [CrossRef] [PubMed] 
13. Asakuma, S.; Hatakeyama, E.; Urashima, T.; Yoshida, E.; Katayama, T.; Yamamoto, K.; Kumagai, H.; Ashida, H.; Hirose, J.; Kitaoka, M. Physiology of Consumption of Human Milk Oligosaccharides by Infant Gut-associated Bifidobacteria. J. Biol. Chem. 2011, 286, 34583-34592. [CrossRef] [PubMed]

14. Sela, D.A.; Garrido, D.; Lerno, L.; Wu, S.; Tan, K.; Eom, H.-J.; Joachimiak, A.; Lebrilla, C.B.; Mills, D.A. Bifidobacterium longum subsp. infantis ATCC $15697 \alpha$-Fucosidases Are Active on Fucosylated Human Milk Oligosaccharides. Appl. Environ. Microbiol. 2011, 78, 795-803. [CrossRef] [PubMed]

15. Locascio, R.G.; Ninonuevo, M.R.; Kronewitter, S.R.; Freeman, S.L.; German, J.B.; Lebrilla, C.B.; Mills, D.A. A versatile and scalable strategy for glycoprofiling bifidobacterial consumption of human milk oligosaccharides. Microb. Biotechnol. 2009, 2, 333-342. [CrossRef] [PubMed]

16. Binia, A.; Lavalle, L.; Chen, C.; Austin, S.; Agosti, M.; Al-Jashi, I.; Pereira, A.B.; Costeira, M.J.; Silva, M.G.; Marchini, G.; et al. Human milk oligosaccharides, infant growth, and adiposity over the first 4 months of lactation. Pediatr. Res. 2021, 90, 684-693. [CrossRef]

17. Sprenger, N.; Lee, L.Y.; De Castro, C.A.; Steenhout, P.; Thakkar, S.K. Longitudinal change of selected human milk oligosaccharides and association to infants' growth, an observatory, single center, longitudinal cohort study. PLoS ONE 2017, 12, e0171814. [CrossRef]

18. Duar, R.M.; Casaburi, G.; Mitchell, R.D.; Scofield, L.N.; Ramirez, C.A.O.; Barile, D.; Henrick, B.M.; Frese, S.A. Comparative Genome Analysis of Bifidobacterium longum subsp. infantis Strains Reveals Variation in Human Milk Oligosaccharide Utilization Genes among Commercial Probiotics. Nutrients 2020, 12, 3247.

19. Smilowitz, J.T.; Moya, J.; Breck, M.A.; Cook, C.; Fineberg, A.; Angkustsiri, K.; Underwood, M.A. Erratum to: Safety and tolerability of Bifidobacterium longum subspecies infantis EVC001 supplementation in healthy term breastfed infants: A phase I clinical trial. BMC Pediatr. 2017, 17, 180. [CrossRef]

20. Ishizeki, S.; Sugita, M.; Takata, M.; Yaeshima, T. Effect of administration of bifidobacteria on intestinal microbiota in low-birthweight infants and transition of administered bifidobacteria: A comparison between one-species and three-species administration. Anaerobe 2013, 23, 38-44. [CrossRef]

21. FDA. Agency Response Letter GRAS Notice No. GRN 758. Lactobacillus helveticus R0052, Bifidobacterium longum subsp. infantis R0033, and Bifidobacterium bifidum R0071 GRAS Notice 758; FDA: Silver Spring, MD, USA, 2018.

22. FDA. Agency Response Letter GRAS Notice No. GRN 950. Bifidobacterium longum subsp. infantis DSM 33361 GRAS Notice 950; FDA: Silver Spring, MD, USA, 2021.

23. Yoon, S.-H.; Ha, S.-M.; Lim, J.; Kwon, S.; Chun, J. A large-scale evaluation of algorithms to calculate average nucleotide identity. Antonie Leeuwenhoek 2017, 110, 1281-1286. [CrossRef]

24. Liu, B.; Zheng, D.; Jin, Q.; Chen, L.; Yang, J. VFDB 2019: A comparative pathogenomic platform with an interactive web interface. Nucleic Acids Res. 2019, 47, D687-D692. [CrossRef]

25. Bortolaia, V.; Kaas, R.S.; Ruppe, E.; Roberts, M.C.; Schwarz, S.; Cattoir, V.; Philippon, A.; Allesoe, R.L.; Rebelo, A.R.; Florensa, A.F.; et al. ResFinder 4.0 for predictions of phenotypes from genotypes. J. Antimicrob. Chemother. 2020, 75, 3491-3500. [CrossRef] [PubMed]

26. Zankari, E.; Allesøe, R.; Joensen, K.G.; Cavaco, L.M.; Lund, O.; Aarestrup, F.M. PointFinder: A novel web tool for WGS-based detection of antimicrobial resistance associated with chromosomal point mutations in bacterial pathogens. J. Antimicrob. Chemother. 2017, 72, 2764-2768. [CrossRef]

27. Feldgarden, M.; Brover, V.; Haft, D.H.; Prasad, A.B.; Slotta, D.J.; Tolstoy, I.; Tyson, G.H.; Zhao, S.; Hsu, C.-H.; McDermott, P.F.; et al. Validating the AMRFinder Tool and Resistance Gene Database by Using Antimicrobial Resistance Genotype-Phenotype Correlations in a Collection of Isolates. Antimicrob. Agents Chemother. 2019, 63, e00483-19. [CrossRef]

28. Wei, Y.-X.; Zhang, Z.-Y.; Liu, C.; Malakar, P.K.; Guo, X.-K. Safety assessment of Bifidobacterium longum JDM301 based on complete genome sequences. World J. Gastroenterol. 2012, 18, 479-488. [CrossRef]

29. Caspi, R.; Billington, R.; Keseler, I.M.; Kothari, A.; Krummenacker, M.; Midford, P.E.; Ong, W.K.; Paley, S.; Subhraveti, P.; Karp, P.D. The MetaCyc database of metabolic pathways and enzymes-A 2019 update. Nucleic Acids Res. 2020, 48, D445-D453. [CrossRef]

30. Austin, S.; Bénet, T. Quantitative determination of non-lactose milk oligosaccharides. Anal. Chim. Acta 2018, 1010, 86-96. [CrossRef]

31. EFSA Panel on Additives and Products or Substances used in Animal Feed (FEEDAP); Rychen, G.; Aquilina, G.; Azimonti, G.; Bampidis, V.; Bastos, M.D.L.; Bories, G.; Chesson, A.; Cocconcelli, P.S.; Flachowsky, G.; et al. Guidance on the characterisation of microorganisms used as feed additives or as production organisms. EFSA J. 2018, 16, e05206. [PubMed]

32. Pightling, A.W.; Pettengill, J.B.; Luo, Y.; Baugher, J.; Rand, H.; Strain, E. Interpreting Whole-Genome Sequence Analyses of Foodborne Bacteria for Regulatory Applications and Outbreak Investigations. Front. Microbiol. 2018, 9, 1482. [CrossRef] [PubMed]

33. Kiwaki, M.; Sato, T. Antimicrobial susceptibility of Bifidobacterium breve strains and genetic analysis of streptomycin resistance of probiotic B. breve strain Yakult. Int. J. Food Microbiol. 2009, 134, 211-215. [CrossRef] [PubMed]

34. Xiao, J.-Z.; Takahashi, S.; Odamaki, T.; Yaeshima, T.; Iwatsuki, K. Antibiotic Susceptibility of Bifidobacterial Strains Distributed in the Japanese Market. Biosci. Biotechnol. Biochem. 2010, 74, 336-342. [CrossRef]

35. Wireman, J.; Liebert, C.A.; Smith, T.; Summers, A.O. Association of mercury resistance with antibiotic resistance in the gramnegative fecal bacteria of primates. Appl. Environ. Microbiol. 1997, 63, 4494-4503. [CrossRef] [PubMed] 
36. Biswas, R.; Halder, U.; Kabiraj, A.; Mondal, A.; Bandopadhyay, R. Overview on the role of heavy metals tolerance on developing antibiotic resistance in both Gram-negative and Gram-positive bacteria. Arch. Microbiol. 2021, 203, 2761-2770. [CrossRef]

37. Sakanaka, M.; Fukiya, S.; Kobayashi, R.; Abe, A.; Hirayama, Y.; Kano, Y.; Yokota, A. Isolation and transposition properties of ISBlo11, an active insertion sequence belonging to the IS3 family, from Bifidobacterium longum 105-A. FEMS Microbiol. Lett. 2015, 362, fnv032. [CrossRef] [PubMed]

38. Milani, C.; Duranti, S.; Lugli, G.A.; Bottacini, F.; Strati, F.; Arioli, S.; Foroni, E.; Turroni, F.; van Sinderen, D.; Ventura, M. Comparative Genomics of Bifidobacterium animalis subsp. lactis Reveals a Strict Monophyletic Bifidobacterial Taxon. Appl. Environ. Microbiol. 2013, 79, 4304-4315. [CrossRef] [PubMed]

39. Morovic, W.; Roos, P.; Zabel, B.; Hidalgo-Cantabrana, C.; Kiefer, A.; Barrangou, R. Transcriptional and Functional Analysis of Bifidobacterium animalis subsp. lactis Exposure to Tetracycline. Appl. Environ. Microbiol. 2018, 84, e1999-18. [CrossRef] [PubMed]

40. EFSA. Outcome of the public consultation on the draft Guidance on the assessment of bacterial susceptibility to antimicrobials of human and veterinary importance. EFSA Supporting Publ. 2012, 9, 316E.

41. van Reenen, C.A.; Dicks, L.M. Horizontal gene transfer amongst probiotic lactic acid bacteria and other intestinal microbiota: What are the possibilities? A review. Arch. Microbiol. 2011, 193, 157-168. [CrossRef]

42. Kim, M.J.; Ku, S.; Kim, S.Y.; Lee, H.H.; Jin, H.; Kang, S.; Li, R.; Johnston, T.V.; Park, M.S.; Ji, G.E. Safety Evaluations of Bifidobacterium bifidum BGN4 and Bifidobacterium longum BORI. Int. J. Mol. Sci. 2018, 19, 1422. [CrossRef]

43. Cohen, K.A.; Stott, K.; Munsamy, V.; Manson, A.L.; Earl, A.M.; Pym, A.S. Evidence for Expanding the Role of Streptomycin in the Management of Drug-Resistant Mycobacterium tuberculosis. Antimicrob. Agents Chemother. 2020, 64, e00860-20. [CrossRef] [PubMed] 